\title{
Magnetic resonance imaging of lower limb muscle injury
}

Hoda Salah Darwish ${ }^{1 *}$ and Mohamed Yasser Habash ${ }^{2}$

*Correspondence: darwish.hoda@yahoo.com

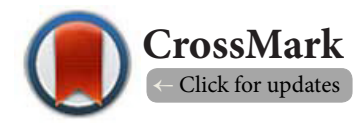

\begin{abstract}
${ }^{1}$ Assistant professor of radio-diagnosis, Faculty of Medicine, Suez Canal University, Egypt, Consultant Radiologist, Dallah Hospital, Riyadh, Kingdom of Saudi Arabia.

${ }^{2}$ Undergraduate, Faculty of Medicine, Cairo University, Egypt.
\end{abstract}

\begin{abstract}
Aim: The aim of this study is to evaluate the role of magnetic resonance imaging (MRI) in the diagnosis of lower limb traumatic muscle injuries.

Methods: From July 2016 to February 2017, MRI study of 46 patients with a history of traumatic muscle injuries to the lower limbs was retrospectively examined. The MRI study was done within a maximum of 5 days of trauma.

Results: All our 46 patients that included in our study were males; 10 out of 46 patients (19\%) had normal study with no MRI evidences of muscle contusion, myotendinous strain, or tendon avulsion. The majority of remaining 37 patients, 28 cases (75\%) showing injury to knee and thigh muscles while 9 cases (24\%) showing ankle \& leg muscles injury. The vastus intermedius muscle was the most susceptible lower limb muscle to injury in our study, $10 / 37$ cases (27\%) followed by the hamstring muscles, semitendinosous, $5 / 37$ cases $(13 \%)$ and biceps femoirs, $3 / 37$ cases (8\%); while the injury at adductor longus muscle was noted at , 3/37 cases $(8 \%)$. Multi-muscles injury was seen in 6/37 cases (16\%). Quadriceps tendon complete tendon avulsion (disruption) was noted in only one case (3\%). A complete rupture of Achilles tendon was found in 1/37 patient (3\%); a partial rupture of the Achilles tendon in $2 / 37$ patients (6\%), another $2 / 37$ cases (6\%) showing avulsion injury to the adductor longus tendon. $1 / 37$ patient (3\%) showing medical gastrocanemious muscles injury and $3 / 37$ cases $(8 \%)$ showing lateral gastrcanemious muscles injury. Associated bone fracture seen in 5 cases (2 cases femoral fracture and 1 cases tibial fractures and 2 cases both tibial and fibular fracture), meniscal tear seen in 5 cases, and ligamentous abnormalities seen in 7 cases $(3$ cases patellar ligament, 3 cases ACL and 2 cases both ACL and PCL )
\end{abstract}

Conclusion: MR imaging is the imaging modality of choice for evaluation of acute traumatic musculotendinous injuries of the lower limb. It can provide useful information about the extent and location of the injury as well as associated bone, meniscal, and ligamentous abnormalities.

Keywords: Muscles, muscles contusion, lower limb muscle injury, magnetic resonance, myotendinous strain, tendon avulsion

\section{Introduction}

Acute skeletal muscle injury is one of the major causative factors for loss of playing time in all athletes. It is caused either by direct trauma, in the form of blunt impact, or indirect trauma following a powerful eccentric muscle contraction, typically at the onset of a sprint movement for hamstring or calf injury and while striking a ball for quadriceps injury [1].

Magnetic resonance (MR) imaging, with its exquisite softtissue contrast resolution, is the imaging modality of choice for evaluation of acute traumatic musculotendinous abnormalities [2].
MR imaging allows optimal assessment of damage to all components of the musculotendinous unit including the muscle belly, myotendinous junction, and tendon insertion site $[3,4]$. In addition, the capability of MR imaging for demonstrating occult muscle trauma makes it a useful tool for assessment of this condition [5].

Three discrete categories of acute injuries to the musculotendinous unit can be defined based on the mechanism of trauma and the site of involvement: muscle contusion, myotendinous strain, and tendon avulsion [2].

Muscle contusions are secondary to direct trauma, usually

(ㄷ) 2017 Darwish et al; licensee Herbert Publications Ltd. This is an Open Access article distributed under the terms of Creative Commons Attribution License (http://creativecommons.org/licenses/by/3.0). This permits unrestricted use, distribution, and reproduction in any medium, provided the original work is properly cited. 
by a blunt object [6]. They usually occur deep in the muscle belly and tend to be less symptomatic than strains, since the latter are commonly located in the superficial muscle layers [5].

Myotendinous strains are induced by indirect trauma from excessive stretching [6]. They are usually the result of a single major traumatic event. Strain injuries affect the myotendinous junction, the weakest point of the musculotendinous unit [7].

Acute avulsion injury at the tendon insertion site results from a forceful, unbalanced, and often eccentric muscle contraction [8]. Loss of function and severe tenderness are frequent symptoms in this condition [2].

The extent of musculotendinous injuries and associated architectural distortion is examined by using axial, coronal, and sagittal images oriented along the longitudinal and short axes of the involved musculotendinous unit [3].

Alterations in water content in the affected musculotendinous units are common to all forms of acute traumatic injuries [3,4].

Short inversion time inversion- recovery (STIR) or fatsuppressed T2- weighted sequences enable detection of edematous changes in the musculotendinous unit and are of paramount importance for diagnosis of these lesions [4-9].

Our article reviews the MRI findings in lower limb acute traumatic musculotendinous injuries. The MR imaging characteristics of muscle contusions, myotendinous strains, and avulsion injuries are emphasized. Associated Intramuscular hematomas bone, meniscal, and ligamentous abnormalities are also described.

\section{Material and Methods}

A total number of 46 patients, whose MRI studies were done from July 2016 to February 2017, were retrieved using picture archiving and communication system and were evaluated retrospectively.

Human ethics committee approval for this study was obtained from the institutional review board of Dallah Hospital, Riyadh, Saudi Arabia.

\section{MRI technique}

All patients underwent MRI using MRI scanner (Optima ${ }^{\mathrm{TM}}$ MR $450 \mathrm{~W} 1.5$ Tesla). The patient is placed comfortably in the supine position to avoid pain, motion and compression of different muscle groups.

Coronal and axial spin-echo T1 weighted [repetition time (TR)/echo time (TE), 650-800 ms/15-20 ms] and fast spin-echo T2 weighted (TR/TE, 3300-4550 ms/90-140 ms) sequences with fat suppression are performed. Alternative to the fatsuppressed T2 weighted sequence is a short-tau inversionrecovery (TR/TE, 3000-3665 ms/15-35 ms; inversion time, 150 $\mathrm{ms}$ ) sequence. Images in a sagittal plane are occasionally acquired to match individual requirements, and intravenous administration of gadolinium- containing contrast material is advised as appropriate.

All images were evaluated on workstation (SECTRA, SWEEDEN).

\section{Statistical analysis}

Data was evaluated by using statistical package for social sciences (SPSS) software version 10 for calculating percentages and frequencies.

\section{Results}

In our study, 46 patients were included. The mean age of the patients was $16 \pm 35$ years, all patients were males.

Out of 46 patients in our study, 10 patients (19\%) had normal study with no MRI evidences of muscle contusion, myotendinous strain, or tendon avulsion.

Sudden onset pain localized to a single muscle occurring during a period of powerful eccentric muscle contraction is the typical presentation of all our cases with muscle strain.

MRI study was performed shortly after the trauma within a maximum of 5 days in all our included cases.

The majority of remaining 37 patients, 28 cases (75\%) showing injury to knee and thigh muscles while 9 cases $(25 \%)$ showing ankle \& leg muscles injury.

The vastus intermedius muscle (Figure 1 ) was the most susceptible to injury, $10 / 37$ cases $(27 \%)$ followed by the hamstring muscles, semitendinosous, $5 / 37$ cases $(13 \%)$ and biceps femoirs, $3 / 37$ cases (8\%) (Figure 2); while the injury at adductor longus muscle was noted at $3 / 37$ cases (8\%) (Figure 3). Multi-muscles injury was seen in $6 / 37$ cases (16\%) (Figure 1 ).

Quadriceps tendon complete tendon avulsion (disruption) was noted in only one case (3\%). It was associated with medial patellar retinaculum laxity causing lateral patellar sublaxation with tortousity of the infrapatellar tendon (patellar ligament) (Figure 4).

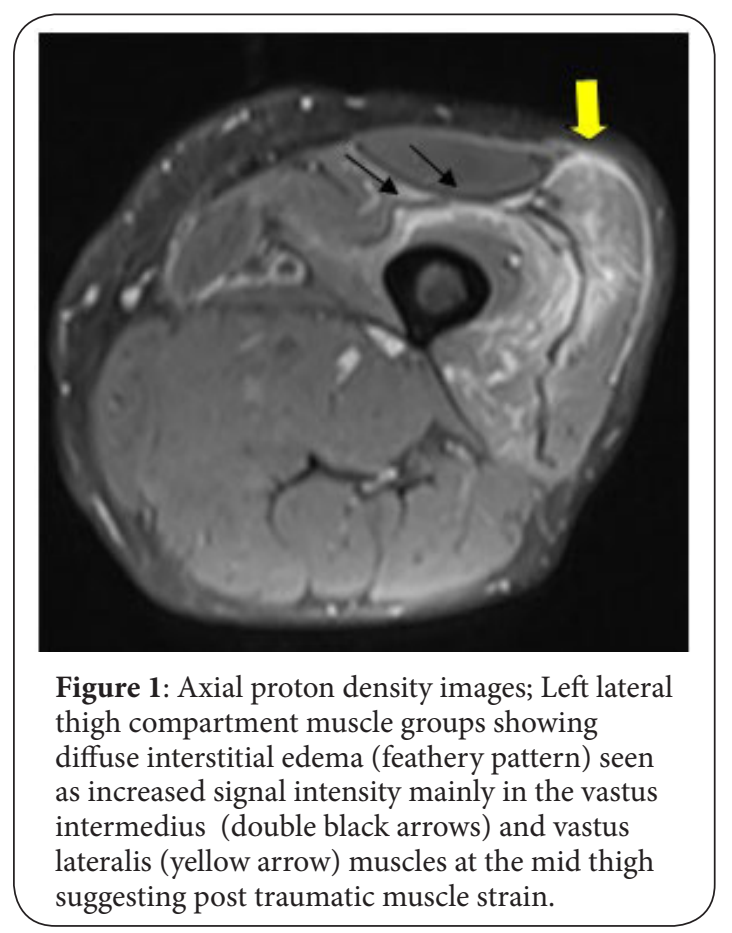




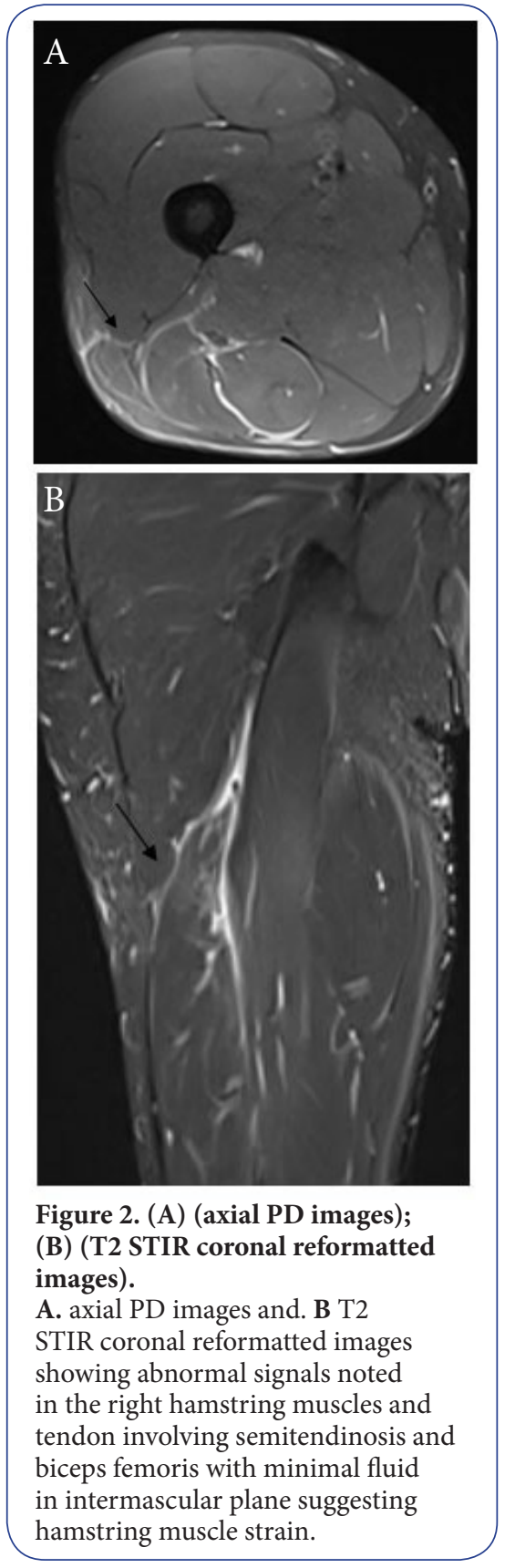

A partial rupture of the Achilles tendon in 2/37 patients (6\%) (Figure 5), A complete rupture of Achilles tendon (Figure 6) found in $1 / 37$ patient ( $3 \%$ ); another $2 / 37$ cases ( $6 \%$ ) showing avulsion injury to the adductor longus tendon (Figure 7 ).

$1 / 37$ patient (3\%) showing medical gastrocanemious muscles injury and $3 / 37$ cases (8\%) showing lateral gastrcanemious muscles injury (Figure 8).

Associated bone fracture seen in only 5 cases ( 2 cases femoral fracture and 1 cases tibial fractures and 2 cases both tibial and fibular fracture), meniscal tear seen in 5 cases, and ligamentous abnormalities was seen in 7 cases ( 3 cases patellar ligament, 3 cases $A C L$ and 2 cases both $A C L$ and $P C L$ ).

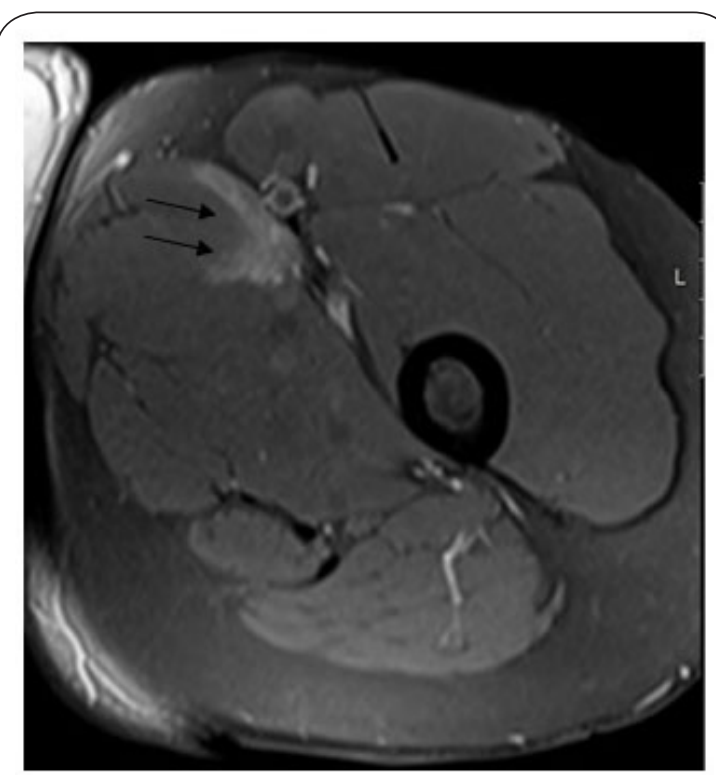

Figure 3. Axial proton density images showing abnormal high signal in the proximal part of the belly of the left adductor longus muscle suggesting muscle strain (arrows).

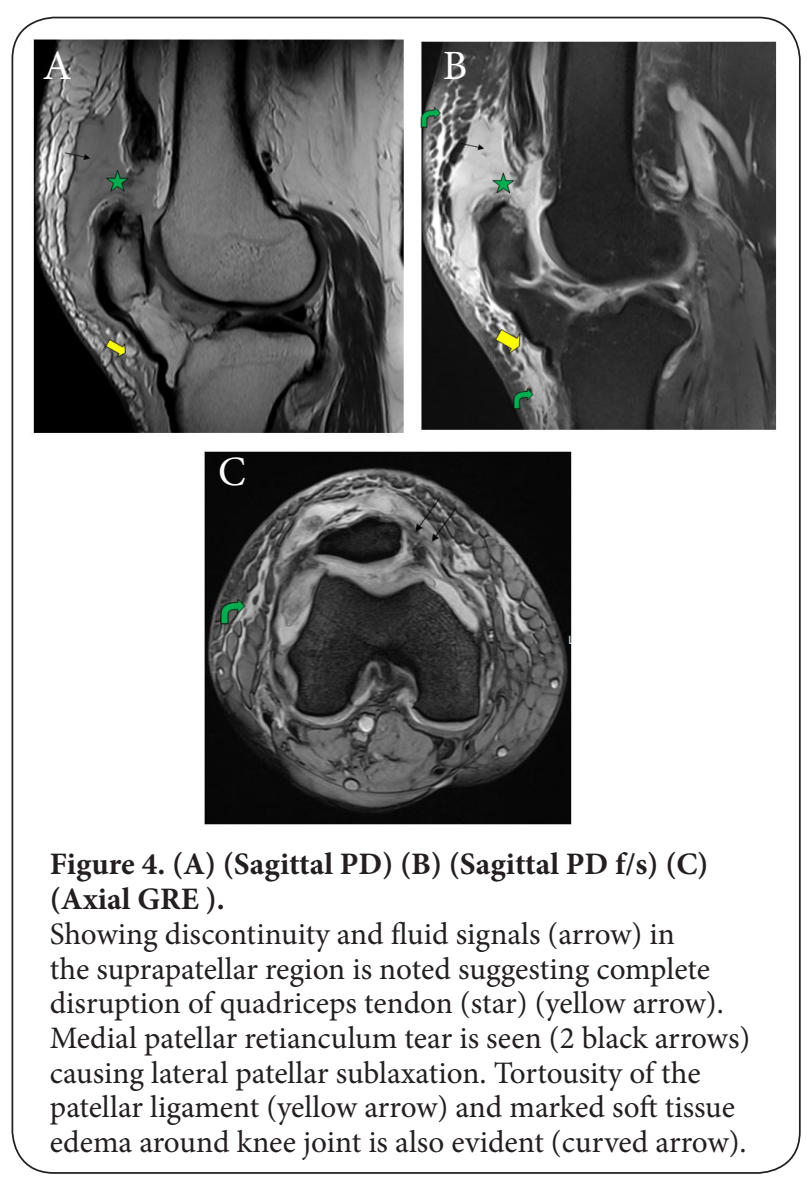

Post traumatic hematomas were seen in about 12 of 37 cases (32\%). 


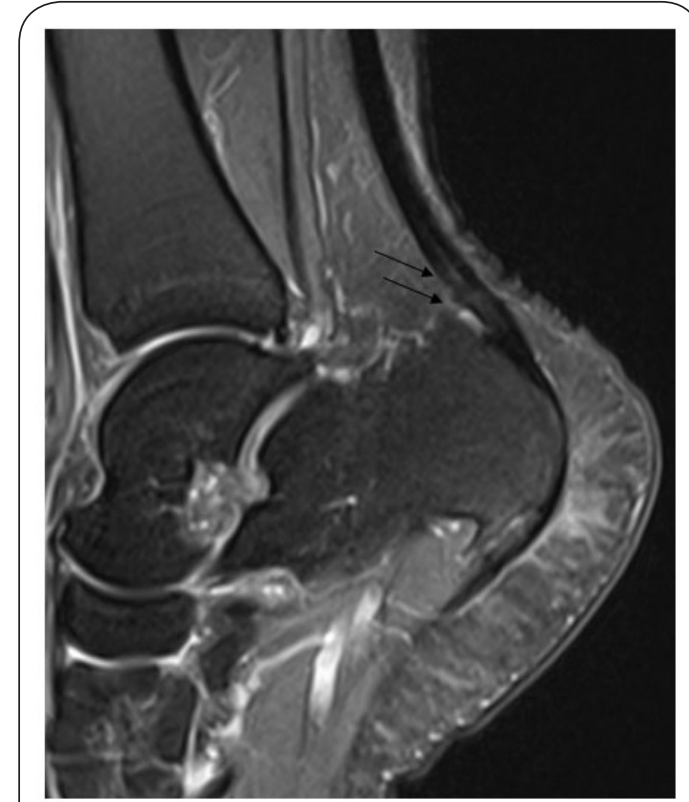

Figure 5. Sagittal PD image showing mild increase in the signal intensity of the lower end of Tendo- Achilles (Double arrows) with subtle fluid seen at retrocalacneal bursa suggesting strain/partial tear of the tendon.

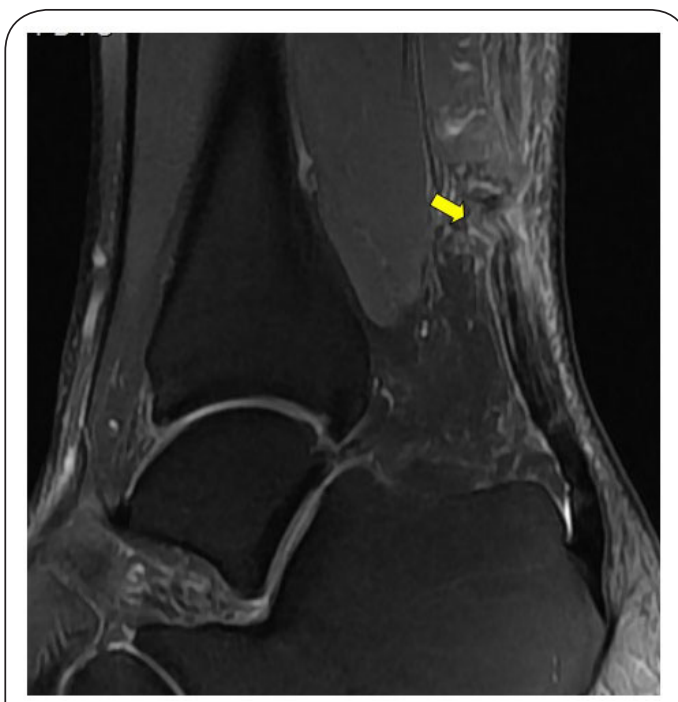

Figure 6. Sagittal PD image showing laxity of Tendo- Achilles with high grade tear at the myotendinous junction (yellow arrow). Few intact fibers are noted at the rupture site suggesting almost complete rupture with few intact fibers.

The haematoma was seen within the muscle (intramuscular hematoma) in 5 cases (Figure 9), between muscles (intermuscular) in 4 cases, and at the muscle-bone interface in 3 cases.

\section{Discussion}

MRI is able to reveal lesions and provide an accurate assess-
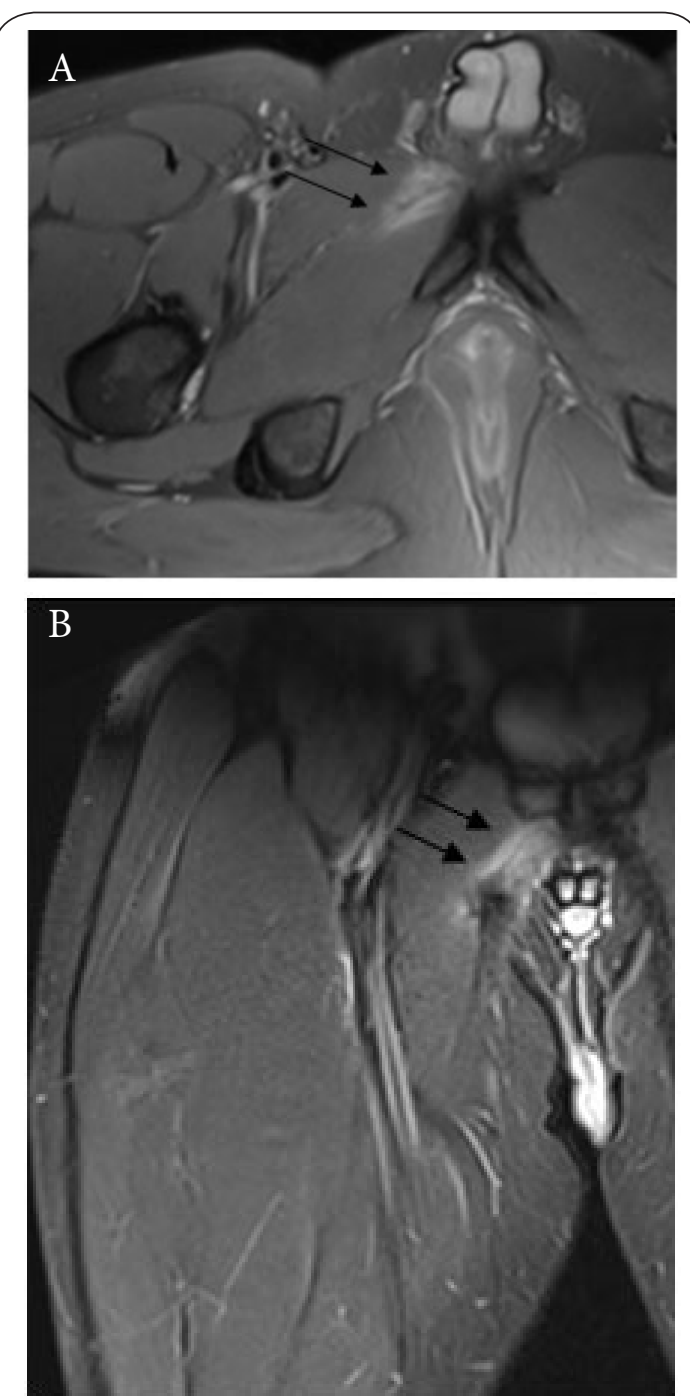

Figure 7. (A) Axial PD and (B) Coronal T1 showing mild edema along with mild fluid signal and discontinuity of some fibers of right adductor longus proximal part at pubic insertion (arrows) with retraction of muscle belly suggesting avulsion injury to the adductor longus tendon from the pubic insertion. ment of site and extent of injury in patients with lower limb traumatic muscle injuries.

We retrospectively studied a total of 46 patients presented to our Radiology department with history of lower limb trauma and clinical suspicion of lower limb muscular injury. MR imaging was carried out using MRI scanner Optima ${ }^{\mathrm{TM}} \mathrm{MR}$ 450 W 1.5 Tesla with phased-array coil.

The quadriceps muscle group extends the knee joint. It is composed of four muscles: the vastus lateralis, vastus medialis, and vastus intermedius, which arise from the proximal femoral shaft, and the rectus femoris, which originates from the anterior inferior iliac spine [10]. 

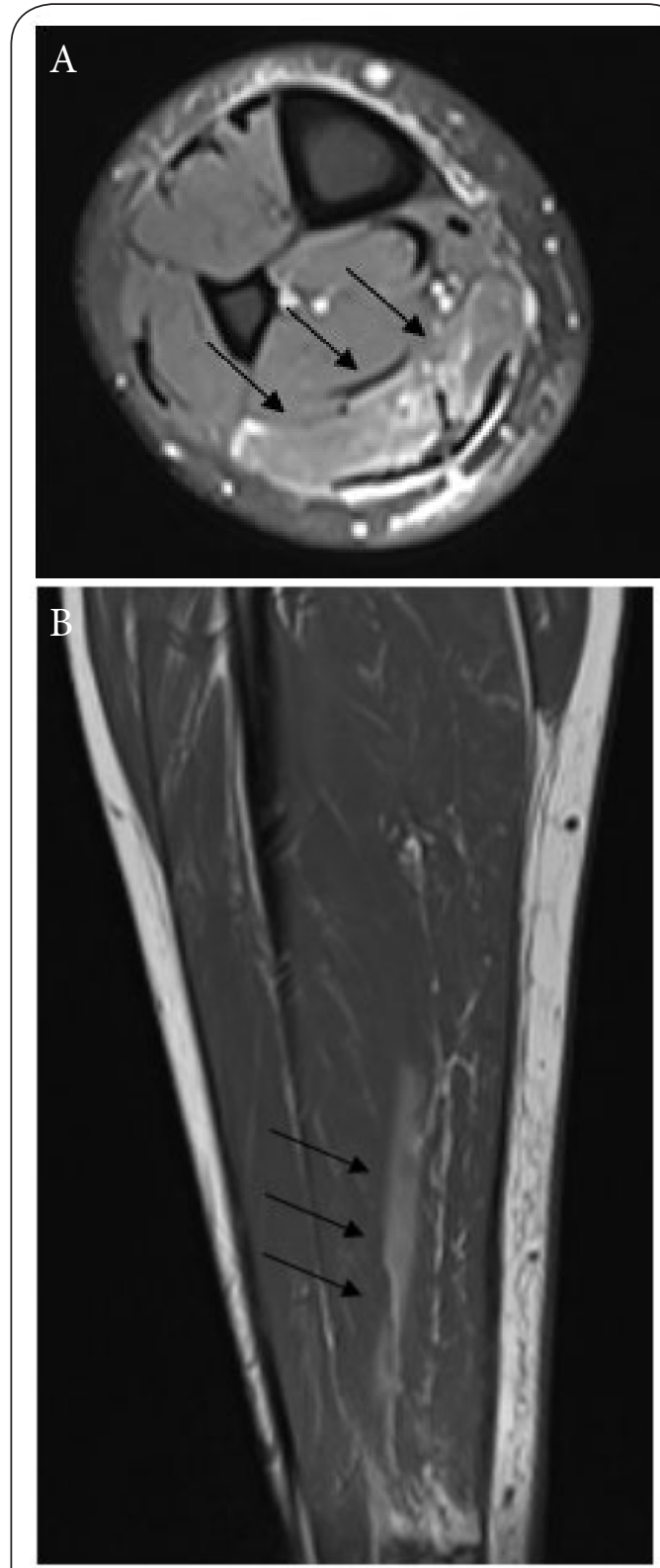

Figure 8. (A) Axial PD image and (B) Coronal T1 weighted image; showing abnormal high signals intensity within the bellies of gastrocnemius muscle suggesting muscle strain. Surrounding soft tissue edema is also noted.

Among the quadriceps muscles, the rectus femoris is the most susceptible to injury at the myotendinous junction [4]. This susceptibility is due to its superficial location, predominance of type II fibers, eccentric muscle action, and extension across two joints [6].

However, in our study, we found that the vastus intermedius muscle is the most commonly injured muscle, 10/37 cases (27\%), it was followed by the hamstring muscles, semitendinosous, $5 / 37$ cases (13\%) and biceps femoris, $3 / 37$ cases ( $8 \%$ ).

In our study as reported with authors, the MR imaging appea-

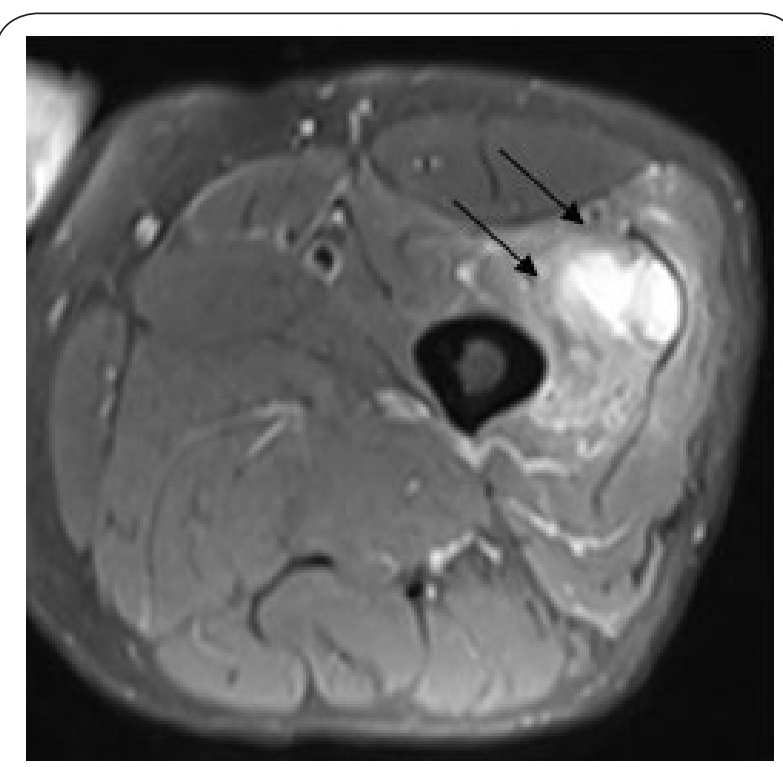

Figure 9. (A) Axial PD and (B) T2 coronal images showing a well defined high signal intensity localized area seen at left vastus intermedius muscle suggesting acute intramuscular hematoma (arrows).

rance of muscle contusions varies according to the severity of the lesion. Diffuse edematous changes of the muscle manifest as a feathery appearance on STIR and T2-weighted images [9].

Myotendinous strains can be classified at MR imaging on the basis of the extent of disruption [4].

First-degree strains imply a minor degree of fiber disruption. Interstitial edema and hemorrhage are present at the myotendinous junction and extend into the adjacent muscle fascicles, producing a feathery appearance on MR images [4-9].

Second-degree injuries are characterized by a partial tear without retraction. A hematoma at the myotendinous junction is frequently observed, as are perifascial fluid collections. While third-degree strains involve a complete rupture of the myotendinous unit; this diagnosis is usually made on clinical grounds. MR imaging is useful when preoperative assessment of the extent of retraction is needed [5].

In our study most of cases showing first degree of muscle strain, 2 cases showing second degree ( 2 cases partial tear of Achilles tendon) while 4 cases showing third degree including 1 case showing complete disruption of the quadriceps and 1 case complete rupture of Achilles tendon and 2 cases avulsion injury to the adductor longus tendon.

The quadriceps tendon has a trilaminar configuration, being formed by a superficial layer from the rectus femoris, a middle layer from the vastus lateralis and medialis, and a deeper layer from the vastus intermedius [11,12].

Acute avulsion injury manifests on MR images as a hematoma and periosteal stripping at the tendon attachment site [13]. Waviness and retraction of the torn end of the tendon along with a fragment of bone or cartilage [14] may also be 
found. In our study quadriceps tendon complete tendon avulsion (disruption) was noted in only one case (3\%). It was manifested as discontinuity and fluid signal in the suprapatellar segment of quadriceps muscle suggesting complete tears of the quadriceps tendon a few centimeters from the upper patellar pole, associated with lateral patellar sublaxation and tortousity of patellar ligament.

Complete disruption of the quadriceps musculotendinous unit at the tendon level often occurs as the end result of repetitive microtrauma or secondary to weakening by other underlying conditions (gout, diabetes, hyperparathyroidism, collagen vascular diseases). Also strong deceleration will result in acute partial or complete tears of the quadriceps [15].

The Achilles tendon, the largest \& strongest tendon in the body, is vulnerable to injury because of its limited blood supply and the combination of forces to which it is subjected. Aging and increased activity (particularly velocity sports) increase the chance of injury to the Achilles tendon [16].

In our study complete rupture of Achilles tendon was found in $1 / 37$ patient (3\%); while a partial rupture of the Achilles tendon in $2 / 37$ patients (6\%).

In our study another $2 / 37$ cases (6\%) showing avulsion injury to the adductor longus tendon from the pubis with retraction of the muscle belly.

Intramuscular hematomas can develop with more severe trauma [5]. In our study the severity of the injury ranges from a small muscle contusion to a large haematoma. Post traumatic hematomas were seen in about 12 of 37 cases (32\%). It was seen within the muscle (intramuscular hematoma) in 5 cases, between muscles (intermuscular) in 4 cases, and at the muscle-bone interface in 3 cases.

The MR imaging characteristics of that hematoma was affected by its age [3]. Acute hematomas in our cases are isointense to skeletal muscle on T1-weighted images and hypointense on T2-weighted or STIR images. However, cases with subacute hematomas demonstrate increased signal intensity on T1-weighted images and heterogeneous signal intensity on T2-weighted images that is due to the presence of extracellular methemoglobin. In the chronic hematoma as the lesion ages, the wall of the hematoma became hypointense from hemosiderin deposition and fibrosis [17].

Some authors reported that, differential diagnosis between muscle contusion and myotendinous strain is difficult based on the MR imaging findings alone [5]. The contusions produce injury at the point of impact, while strains lead to injury at or near the myotendinous junction. Contusions frequently have a diffuse feathery appearance with increased signal intensity on T2-weighted and STIR images, similar to a first-degree myotendinous strain [9]. If fiber disruption occurs, contusions may manifest as an intramuscular hematoma. Contusion hematomas are often located deeper in the muscle belly than hematomas associated with second- and third-degree myotendinous strains [5].

\section{Conclusion}

MRI plays a central role in delineating muscle anatomy and morphology, and providing characterization of muscle composition and its alterations. It is the imaging modality of choice for evaluation of acute traumatic musculotendinous injuries of the lower limb. It can provide useful information about the extent and location of the injury as well as associated bone, meniscal, and ligamentous injuries.

\section{Competing interests}

The authors declare that they have no competing interests. Authors' contributions

\begin{tabular}{|l|c|c|}
\hline Authors' contributions & HSD & MYH \\
\hline Research concept and design & $\checkmark$ & -- \\
\hline Collection and/or assembly of data & $\checkmark$ & -- \\
\hline Data analysis and interpretation & $\checkmark$ & -- \\
\hline Writing the article & $\checkmark$ & $\checkmark$ \\
\hline Critical revision of the article & $\checkmark$ & $\checkmark$ \\
\hline Final approval of article & $\checkmark$ & $\checkmark$ \\
\hline Statistical analysis & $\checkmark$ & $\checkmark$ \\
\hline
\end{tabular}

\section{Acknowledgement}

The authors would like to thank their colleagues for the support and patients for their trust.

\section{Publication history}

Senior Editor: Domenico Rubello, Santa Maria della Misericordia Hospital, Italy.

Received: 29-Mar-2017 Final Revised: 23-May-2017

Accepted: 22-Jun-2017 Published: 03-Jul-2017

\section{References}

1. Lee J and Heal J. Imaging of lower limb muscle injury. Sports radiology. 142-147.

2. Bencardino JT, Rosenberg ZS, Brown RR, Hassankhani A, Lustrin ES and Beltran J. Traumatic musculotendinous injuries of the knee: diagnosis with MR imaging. Radiographics. 2000; 20 Spec No:S103-20. | Article | PubMed

3. Shellock FG, Mink J and Deutsch AL. MR imaging of muscle injuries. Appl Radiol. 1994; 11-18.

4. Palmer WE, Kuong SJ and Elmadbouh HM. MR imaging of myotendinous strain. AJR Am J Roentgenol. 1999; 173:703-9. | Article | PubMed

5. McNamara MT, Greco A. Miscellaneous muscle lesions. In: Fleckenstein JL, Crues JV III and Reimers CD, eds. Muscle imaging in health and disease. New York, NY: Springer-Verlag. 1996; 425-449.

6. Mallone TR. Basic science of musculotendinous structure. In: Garrett WE Jr, Duncan PW, Mallone TR, eds. Muscle injury and rehabilitation. Baltimore, Md: Williams \& Wilkins. 1988; 1-42.

7. Noonan TJ and Garrett WE, Jr. Injuries at the myotendinous junction. Clin Sports Med. 1992; 11:783-806. I PubMed

8. Stevens MA, El-Khoury GY, Kathol MH, Brandser EA and Chow S. Imaging features of avulsion injuries. Radiographics. 1999; 19:655-72. | Article | PubMed 
9. Steinbach LS, Fleckenstein JL and Mink JH. Magnetic resonance imaging of muscle injuries. Orthopedics. 1994; 17:991-9. | PubMed

10. Jenkins DB. Hollinshead's functional anatomy of the limbs and back. Philadelphia, Pa: Saunders, 1991; 233-305.

11. Zeiss J, Saddemi SR and Ebraheim NA. MR imaging of the quadriceps tendon: normal layered configuration and its importance in cases of tendon rupture. AJR Am J Roentgenol. 1992; 159:1031-4. | Article | PubMed

12. Sonin AH. Magnetic resonance imaging of the extensor mechanism. Magn Reson Imaging Clin N Am. 1994; 2:401-11. | PubMed

13. Connell DA, Potter HG, Sherman MF and Wickiewicz TL. Injuries of the pectoralis major muscle: evaluation with MR imaging. Radiology. 1999; 210:785-91. | Article | PubMed

14. Bates DG, Hresko MT and Jaramillo D. Patellar sleeve fracture: demonstration with MR imaging. Radiology. 1994; 193:825-7. I Article | PubMed

15. Sonin AH. Magnetic resonance imaging of the extensor mechanism. Magn Reson Imaging Clin N Am. 1994; 2:401-11. I PubMed

16. Sonin AH, Fitzgerald SW, Bresler ME, Kirsch MD, Hoff FL and Friedman $\mathrm{H}$. MR imaging appearance of the extensor mechanism of the knee: functional anatomy and injury patterns. Radiographics. 1995; 15:367-82. | Article | PubMed

17. Mazzone MF and McCue T. Common conditions of the achilles tendon. Am Fam Physician. 2002; 65:1805-10. | Article | PubMed

18. De Smet AA, Fisher DR, Heiner JP and Keene JS. Magnetic resonance imaging of muscle tears. Skeletal Radiol. 1990; 19:283-6. | PubMed

\section{Citation:}

Darwish HS and Habash MY. Magnetic resonance imaging of lower limb muscle injury. Med Imaging and Radiol. 2017; 5:2.

http://dx.doi.org/10.7243/2054-1945-5-2 